\title{
Image processing-based model for tortuosity measurement of retinal blood vessels
}

Sanyukta Chetia, S.R. Nirmala

Department of Electronics and Communication Engineering, Gauhati University, Guwahati, Assam, India

\section{Abstract}

Purpose: The study of retinal blood vessel morphology is of great importance in retinal image analysis. The retinal blood vessels have a number of distinct features such as width, diameter, tortuosity, etc. In this paper, a method is proposed to measure the tortuosity of retinal blood vessels obtained from retinal fundus images. Tortuosity is a situation in which blood vessels become tortuous, that is, curved or non-smooth. It is one of the earliest changes that occur in blood vessels in some retinal diseases. Hence, its detection at an early stage can prevent the progression of retinal diseases such as diabetic retinopathy, hypertensive retinopathy, retinopathy of prematurity, etc. The present study focuses on the measurement of retinal blood vessel tortuosity for the analysis of hypertensive retinopathy. Hypertensive retinopathy is a condition in which the retinal vessels undergo changes and become tortuous due to long term high blood pressure. Early recognition of hypertensive retinopathy signs remains an important step in determining the target-organ damage and risk assessment of hypertensive patients. Hence, this paper attempts to estimate tortuosity using image-processing techniques that have been tested on artery and vein segments of retinal images.

Design: Image processing-based model designed to measure blood vessel tortuosity. Methods: In this paper, a novel image processing-based model is proposed for tortuosity measurement. This parameter will be helpful for analyzing hypertensive retinopathy. To test the effectiveness of the system in determining tortuosity, the method is first applied on a set of synthetically generated blood vessels. Then, the method is repeated on blood vessel (both artery and vein) segments extracted

Correspondence: S.R. Nirmala, Department of Electronics and Communication Engineering, Gauhati University, Assam, India. E-mail: nirmalasr3@gmail.com 
from retinal images collected from publicly available databases and on images collected from a local eye hospital. The blood vessel segment images that are used in the method are binary images where blood vessels are represented by white pixels (foreground), while black pixels represent the background. Vessels are then classified into normal, moderately tortuous, and severely tortuous by following the analysis performed on the images in the Retinal Vessel Tortuosity Data Set (RET-TORT) obtained from Biolm Lab, Laboratory of Biomedical Imaging (Padova, Italy). This database consists of 30 artery segments and 30 vein segments, which were manually ordered on the basis of increasing tortuosity by Dr. S. Piermarocchi, a retinal specialist belonging to the Department of Ophthalmology of the University of Padova (Italy). The artery and vein segments with the fewest number of turns are given a low tortuosity ranking, while those with the greatest number of turns are given a high tortuosity ranking by the expert. Based on this concept, our proposed method defines retinal image segments as normal when they present the fewest number of twists/turns, moderately tortuous when they present more twists/turns than normal but fewer than severely tortuous vessels, and severely tortuous when they present a greater number of twists/turns than moderately tortuous vessels. On implementing our image processing-based method on binary blood vessel segment images that are represented by white pixels, it is found that the vessel pixel (white pixels) count increases with increasing vessel tortuosity. That is, for normal blood vessels, the white pixel count is less compared to moderately tortuous and severely tortuous vessels. It should be noted that the images obtained from the different databases and from the local hospital for this experiment are not hypertensive retinopathy images. Instead, they are mostly normal eye images and very few of them show tortuous blood vessels.

Results: The results of the synthetically generated vessel segment images from the baseline for the evaluation of retinal blood vessel tortuosity. The proposed method is then applied on the retinal vessel segments that are obtained from the DRIVE and HRF databases, respectively. Finally, to evaluate the capability of the proposed method in determining the tortuosity level of the blood vessels, the method is tested with a standard tortuous database, namely, the RET-TORT database. The results are then compared with the tortuosity level mentioned in the database. It was found that our method is able to classify blood vessel images as normal, moderately tortuous, and severely tortuous, with results closely matching the clinical ordering provided by the expert in the RET-TORT database. Subjective evaluation was also performed by research scholars and postgraduate students to cross-validate the results.

Conclusion: The close correlation between the tortuosity evaluation using the proposed method and the clinical ordering of retinal vessels as provided by the retinal specialist in the RET-TORT database shows that, although simple, this method can evaluate the tortuosity of vessel segments effectively. 
Keywords: blood vessel pixels, hypertensive retinopathy, retinal blood vessels, tortuosity

\section{Introduction}

The retina, which forms the third and inner coating of the eye, contains arteries and veins. These retinal blood vessels can be easily imaged non-invasively using a fundus camera. ${ }^{1}$ Retinal vessels have a number of observable features such as width, diameter, tortuosity, etc. These blood vessels are part of the circulatory system that supplies oxygen and nutrients throughout the body. The arteries carry oxygenated blood away from the heart to the body, while veins carry deoxygenated blood from the body to the heart. Thus, the retinal arteries supply oxygenated blood to the retina, while the retinal veins carry away oxygen-poor blood from the retina. Hence, proper functioning of arteries and veins is essential for a healthy retina; changes in retinal vessel features can lead to an unhealthy retina. The pathophysiological changes of these features may be associated with retinopathies or cardiovascular disease. $^{2}$

The term retinopathy refers to damage to the retina. Hypertensive retinopathy is a condition in which the retinal vessels undergo changes due to high blood pressure. ${ }^{3}$ The various vascular signs that arise from elevated blood pressure include arteriolar narrowing, arteriovenous nicking, cotton-wool spots, hard exudates, and optic disc swelling. ${ }^{4}$ Individuals with elevated blood pressure often develop an abnormal curvature along the vessel length, which is referred to as tortuosity. ${ }^{5}$ Generally, in individuals with normal blood pressure $(120 / 80 \mathrm{mmHg})$, blood vessels have smooth inner walls and blood flows through them without any trouble. However, when this pressure rises, i.e., in cases of hypertension $(140 / 90 \mathrm{mmHg}$ or higher over a number of weeks), blood flows through the vessels with a large force, damaging blood vessel walls in such a way that they become thick and non-smooth. This, in turn, causes blood vessels to take on twisted paths, giving rise to tortuosity. Hence, in cases of acute hypertension, there is an increase in vascular tortuosity. ${ }^{6}$ Figures $1 \mathrm{a}$ and $1 \mathrm{~b}$ show a retinal image with normal blood vessels and tortuous blood vessels, respectively. The normal blood vessels are smooth, whereas the tortuous blood vessels are not smooth in appearance. Thus, a blood vessel is said to be tortuous when it presents several twists and turns instead of being straight or gently curved. ${ }^{7}$

Hypertension leads to a number of complications. Hypertensive retinopathy is considered to be one such major complication. A person with hypertensive retinopathy may also develop other complications of hypertension, such as cardiovascular risk and nephropathy. ${ }^{8}$ Hypertensive retinopathy is also associated with congestive heart failure, cerebrovascular disease, and stroke mortality. $\mathrm{A}$ study showed that hypertensive retinopathy is linked to coronary heart disease. ${ }^{9}$ In a population-based study among individuals with hypertension, hyperten- 


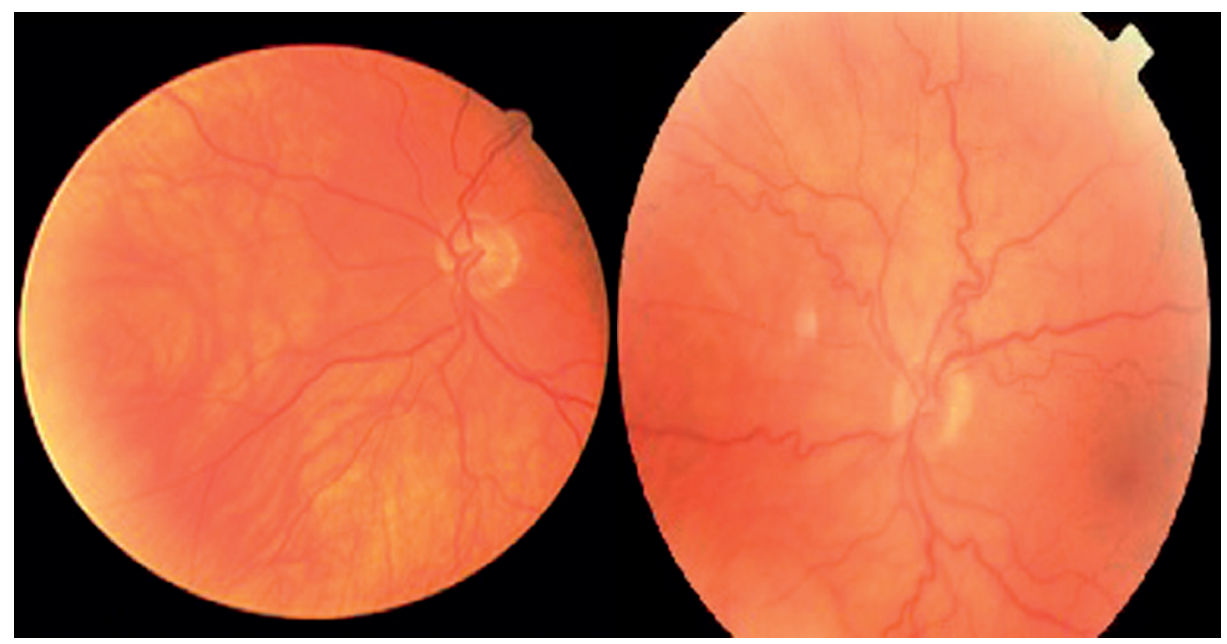

Fig. 1. Retinal fundus images with $(a)$ normal blood vessels and $(b)$ tortuous blood vessels.

sive retinopathy was related to an elevated risk of stroke. Thus, for hypertensive individuals, retinal examination can provide necessary information to determine the risk of stroke. ${ }^{10}$ Retinal examination of hypertensive patients shows that vessel tortuosity increases in case of long-term acute hypertension. Hence, in this work, we present a method based on image processing techniques for measuring retinal blood vessel tortuosity. The proposed method considers both retinal arteries and veins for the purpose of evaluating tortuosity.

\section{Literature review}

Different methods have been proposed to assess retinal vessel tortuosity. Sharbaf et al. proposed a curvature-based algorithm for automatic grading of retinal vessel tortuosity. ${ }^{11}$ The algorithm was applied to evaluate tortuosity in single vessels as well as in a vessel network. To measure tortuosity, the authors used the concept of curvature, which is a mathematical measure for how inflected a curve is at a certain coordinate. The method used for calculating curvature is the template disc method. The basic principle of curvature calculation using this method is to relate the area between the curve and a template disc of a suitable radius of curvature, as shown in Figure 2. To calculate curvature at a point $p(x, y)$, initially a template disc of radius $b$ is drawn around the point. Then the center of the Cartesian coordinate system is set on the point. In the figure, $f(x)$ is a planar curve representing the blood vessel, $A$ is the area between the curve and the template disc of radius $b, \theta_{c}$ is the cross-over angle between the template disc and the cross-over point $\left(\mathrm{k}_{c p}\right)$ with respect to the $x$-axis. The authors have defined the curvature as $k=1 / A^{2}$. Thus, for a large area, 


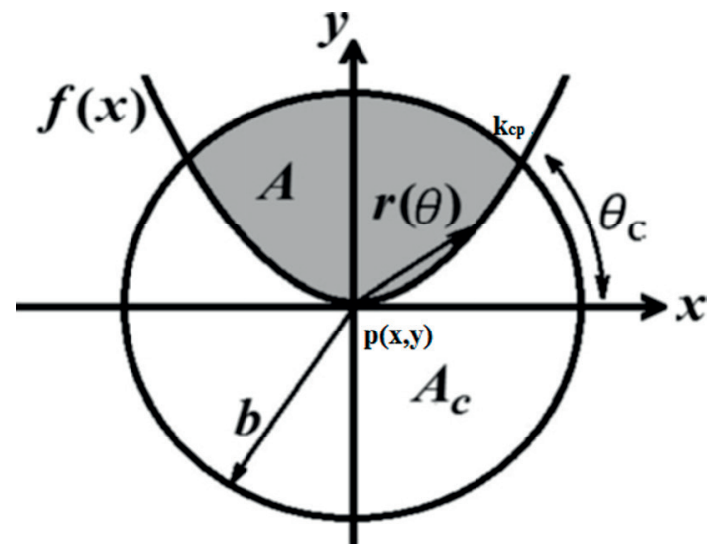

Fig. 2. Curvature calculation using the template disc method.
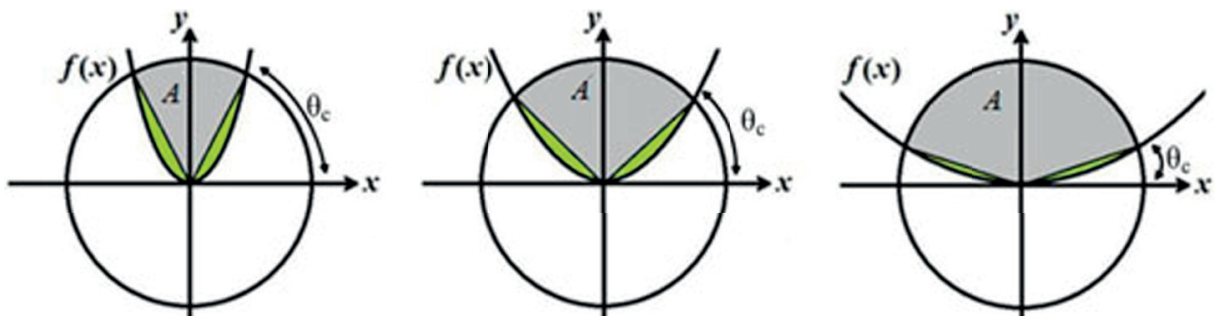

Fig. 3. Approximating area $A$ using the cross-over point of the curve and the template disc.

the curvature is small and vice versa, as shown in Figure 3. After calculating the curvature using the above equation for a single vessel, tortuosity is evaluated for the vessel network. The authors segmented the blood vessels, generated the vascular skeleton map, and evaluated the tortuosity using the formula:

$$
\tau=\frac{1}{\mathrm{~m}} \sum_{i=1}^{m} \mathrm{k}_{i}
$$

where $k_{i}$ denotes curvature for the $i^{\text {th }}$ point on the vessel map and $m$ denotes the total number of points for which curvature is measured. The authors used three databases to find the correlation between their algorithm and the results obtained for these databases. For evaluating tortuosity in a single vessel, the authors used the RET-TORT dataset obtained from Biolm Lab, Laboratory of Biomedical Imaging. ${ }^{12}$ This database was introduced by Grisan et al. ${ }^{13}$ and comprises 30 artery segments and 30 vein segments from normal and hypertensive patients, arranged in order of increasing tortuosity by an expert. In this case, the correlation between the ranking obtained by the algorithm developed by the authors and the expert's ranking is 0.94. The second dataset was provided by Khatam-Al-Anbia Hospital, Mashhad 
(Iran), consisting of ten full retinal images diagnosed with diabetic retinopathy and used for measuring vessel network tortuosity. Based on tortuosity, the images in this dataset are arranged by many experienced experts. Here, the correlation between the method introduced by the authors and the subjective results is 0.95 . The third database comprised a private databank containing 120 full retinal images diagnosed with retinopathy of prematurity (ROP), which was also used to measure vessel network tortuosity. The authors evaluated the performance of their algorithm and obtained a correlation of 0.7 in this case.

Cavallari et al. ${ }^{14}$ proposed a semi-automated, computer-based method for automatic analysis of retinal images in hypertensive retinopathy. The authors defined the tortuosity index as a measure of blood vessel tortuosity. They considered 16 subjects with hypertensive retinopathy and fundoscopy was carried out using a digital fundus camera. Using a software called Cioran, the authors calculated the tortuosity index following three steps:

1. vessel extraction;

2. vessel tracking; and

3. measurement of tortuosity index.

The vessel path is automatically extracted by the process of iterative runs of the subtract background task. On selecting the starting and end points of the vessel segment by the operator, Cioran automatically tracks the vessel. Lastly, for the measurement of tortuosity index, the Bezier and Spline interpolation method is used. Thus, the vessel path is described by a regular analytical function $y=f(x)$. The operator selected start and end points were set to $y=0$. The computation of tortuosity index takes into consideration:

1. the area under the curve described by the segment of the vessel; and

2. the number of times directional changes occur along the vessel path. The directional changes correspond to the points where the first derivative of the analytic function is equal to zero and the vessel path changes its slope.

Turior et $a l .^{15}$ have proposed curvature-based tortuosity metrics in the retinal blood vessels of premature infants. Tortuosity is evaluated by estimating the curvature of each point over some region and then adding the curvature at every pixels of the vessel. The curvature at each point is calculated by proper pairing of chain codes and related chain code rules. The chain code representation is based on certain geometric concepts that are related with the straightness or non-straightness of a digital curve. Figure 4 depicts a segment of a digital curve showing pair of chain codes for $k=8$. The discrete curvature around the concerned point $p_{i}$ is obtained by using the expression:

$$
\begin{aligned}
& \mathrm{k}\left(p_{i}, \mathrm{k}\right)=\frac{1}{k} \sum_{j=1}^{k} \min \left\{\min \left(f_{i+j}^{\prime}, 8-f_{i+j}^{\prime}\right), \min \left(f_{i+j}^{\prime(+1)}, 8-f_{i+j}^{\prime(+1)}\right), \min \left(f_{i+j}^{\prime(-1)}, 8-f_{i+j}^{\prime(-1)}\right)\right\}, \\
& \text { where } \\
& f_{i+j}^{\prime}=\left|f_{i+j}-f_{i-j+1}\right|, \\
& f_{i+j}^{('+1)}=\left|f_{i+j+1}-f_{i-j+1}\right|,
\end{aligned}
$$




$$
f_{i+j}^{\prime(-1)}=\left|f_{i+j}-f_{i-j}\right|
$$

where $f_{i+j}$ and $f_{i-j}$ are the chain code of the $j^{\text {th }}$ leading and following point with respect to the point of interest $p_{i}$, respectively, is an integer given by $f=0,1,2, \ldots$, and $k$ is the number of points used for calculating curvature. After calculating the curvature, the authors estimated the tortuosity of blood vessel as:

$$
\tau=\left(\frac{n_{i c}-1}{n_{i c}}\right) \frac{1}{L} \sum_{i=1}^{n} \mathrm{k}\left(p_{i}, \mathrm{k}\right)
$$

$n_{i c}$ and $\mathrm{L}$ are the number of inflection points and arc length, respectively, and $n$ is the number of pixels in the vessel segment considered. The authors used the Naive Bayes neural classifier to classify non-tortuous, semi-tortuous, or extreme tortuous vessels on 45 retinal images. The retinal images were obtained from the Digital Imaging Research Centre of Kingston University (London, UK), Department of Ophthalmology of the Imperial College (London, UK) and Thammasat Hospital (Thailand). Following this, the authors achieved the best sensitivity and classification rate of $97.8 \%$ and $93.6 \%$ for non-tortuous, semi-tortuous, and extreme tortuous vessel segments, respectively.

El Abbadi and Al Saadi ${ }^{16}$ proposed a method for automatic human retinal vessel tortuosity measurement. The authors used a mask filter to trace the vessel along its course; tortuosity was then evaluated using the arc-to-chord ratio. A $3 \times 3$ mask (Fig. 5) is created and used to calculate vessel segment tortuosity. The center of the mask is placed over the first pixel of the blood vessel segment. The mask is then moved along the complete length of the vessel segment to determine its length.

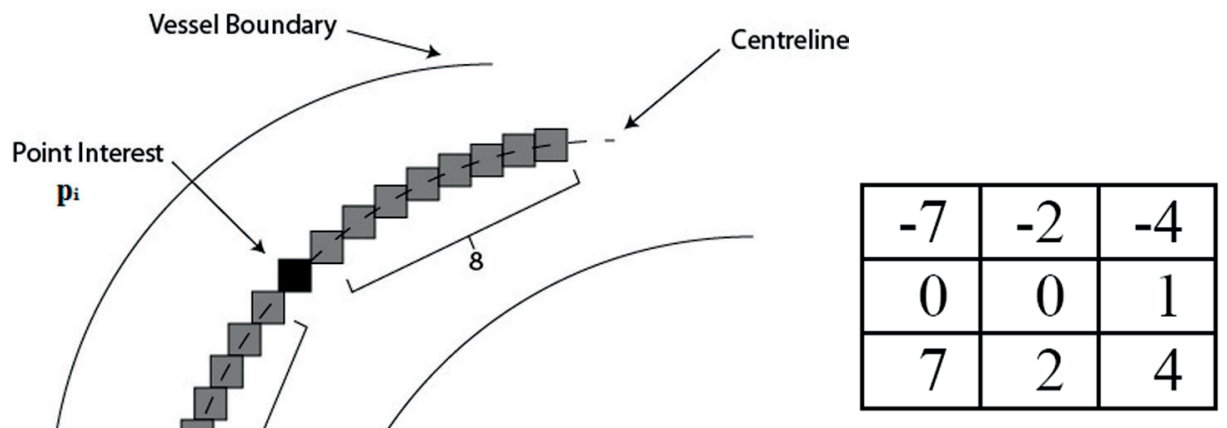

Fig. 5. A $3 \times 3$ mask used for evaluating tortuosity.

Fig. 4. A segment of a digital curve showing pair of chain codes for $k=8$. 

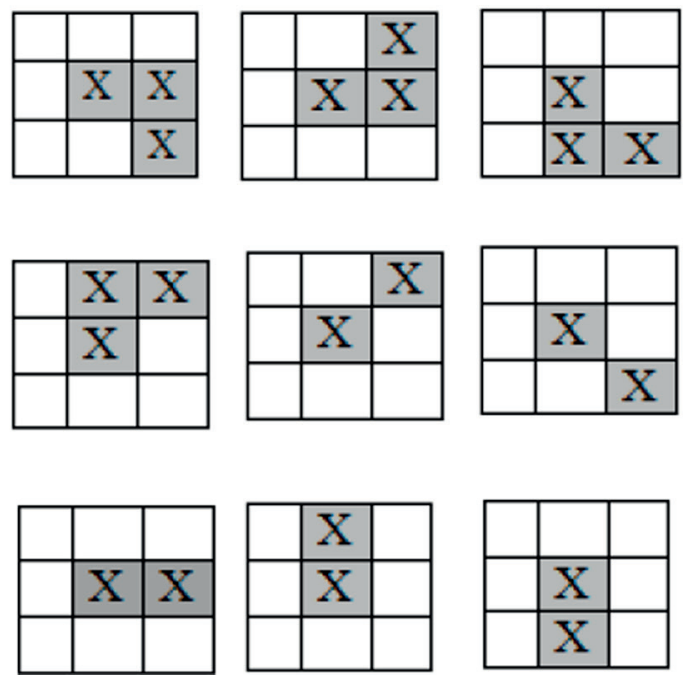

Fig. 6. Probable orientation of the blood vessel from the mask.

The movement of the mask from left to right also provides its orientation. There are nine possible orientations of the blood vessel segment pixels according to the filter. Figure 6 shows the orientation of the blood vessel segment using the $3 \times 3$ mask. In Figure 6 , the blood vessel segment pixels are represented in gray, whereas white represents the background.

Then, using the Euclidean distance, the straight distance between the first and last points of the vessel is calculated as:

$$
\mathrm{D}_{\text {straight }}=\sqrt{\left(x_{N}-x_{1}\right)^{2}+\left(y_{N}-y_{1}\right)^{2}}
$$

where, $\left(x_{1}, y_{1}\right)$ are the coordinates of the first point and $\left(x_{N}, y_{N}\right)$ are the coordinates of the end point of the vessel segment, respectively.

The arc length $\mathrm{L}_{\text {arc }}$ when the mask reaches the last pixel in the blood vessel segment is obtained by adding the distance between consecutive points in the vessel. Mathematically:

$$
\mathrm{L}_{\mathrm{arc}}=\sqrt{\left(\mathrm{x}_{\mathrm{i}+1}-\mathrm{x}_{\mathrm{i}}\right)^{2}+\left(\mathrm{y}_{\mathrm{N}}-\mathrm{y}_{\mathrm{i}}\right)^{2}}
$$

where $\left(x_{i}, y_{i}\right)$ are the coordinates of the $i^{\text {th }}$ pixel in the vessel segment consisting of $\mathrm{N}$ constituent points.

Finally, tortuosity is calculated using the formula:

$$
\text { Tortuosity }=\frac{L_{\text {arc }}}{D_{\text {straight }}}
$$


After a detailed survey of the available literature, we found that several authors have proposed different methods for evaluating tortuosity. However, there is ample room for contributions on evaluating tortuosity in hypertensive retinopathy images. Early detection of this disease is crucial to avoid its related risks. The current trend for tortuosity evaluation is based on manual examination of fundus photographs by human experts. Thus, there is a need to develop a method that could assist experts in determining tortuosity automatically, which is the purpose of the present work. This paper presents an image processing-based method for evaluating retinal blood vessel tortuosity automatically.

\section{Methods}

A block diagram of our proposed method is shown in Figure 7.

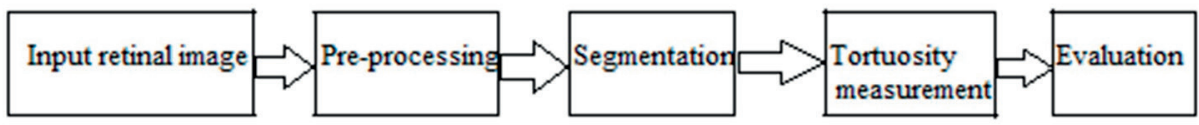

Fig. 7. Block diagram of the proposed model for tortuosity evaluation.

\subsection{Input retinal images}

A total of 55 retinal images are considered in the present work. Of these 55 images, 20 images were collected from the publicly available DRIVE database, ${ }^{17} 30$ images were collected from the publicly available High-Resolution Fundus (HRF) database, ${ }^{18}$ and 5 images were collected from a local eye hospital, the Chandraprabha Eye Hospital (Jorhat, Assam, India). Figure 8 a shows a retinal image, obtained from the DRIVE database, used as input in the proposed method.

\subsection{Pre-processing}

After acquiring the retinal image, the first stage is pre-processing the image. The main function of this step is to obtain uniform illumination in all retinal images. After the pre-processing stage, the images are more suitable for use in the later stages. Pre-processing consists of three main steps: green channel extraction, image complement and histogram equalization.

1. Green channel extraction: The input retinal images from the different databases and from the local hospital are all color images. The color images consist of three main color bands: red (R), green (G), and blue (B), known as RGB images. However, the green band is extracted from this RGB image as it provides a good contrast between the blood vessels and the background (Fig. 8b).

2. Image complement: The green band image is turned into a negative image 

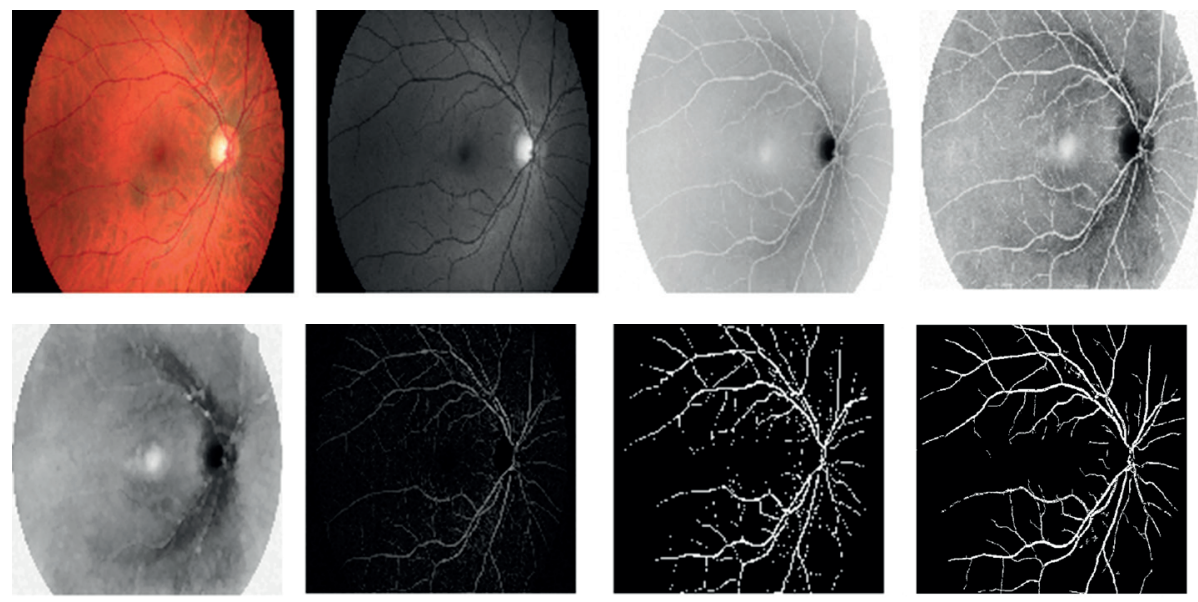

Fig. 8. Results of pre-processing and segmentation: $(a)$ original image, $(b)$ green channel image, $(c)$ complement image, $(d)$ CLAHE image, $(e)$ opened image, $(f)$ disc removed image, and $(g)$ detected blood vessels $(h)$ after removing small non-vessel regions.

in order to make the light areas become darker and vice versa for further processing (Fig. 8c).

3. Histogram equalization:An image enhancement technique known as Contrast Limited Adaptive Histogram Equalization (CLAHE) is used to enhance image contrast. The image is divided into a number of small, non-overlapping regions called tiles and the local contrast of all the regions is enhanced. ${ }^{19}$ The contrast enhanced image is shown in Figure 8d.

\subsection{Segmentation}

The next step after pre-processing is the segmentation of the histogram equalized retinal image. The main function of this step is to extract the blood vessels given that they are the focus of the proposed method. It consists of two main steps: morphological opening and image binarization.

1. Morphological opening: Erosion and dilation are the two basic morphological operations. ${ }^{20}$ When pixels are added to the object boundaries, the object expands; this process is known as dilation. When pixels are removed from the object boundaries, it is known as erosion, which leads to shrinkage of objects. Here, a disc-shaped structuring element of radius 19 is used to remove objects, such as the optic disc, from the retinal image. For this, erosion is performed first, followed by dilation operation. Figure 8e depicts the result of the opening operation. A disc-removed image is then obtained by subtracting the opened image from the CLAHE image (Fig. 8f).

2. Image binarization: Image binarization is the process of creating a binary image with only two sets of pixel values: white as foreground and black as 
background, or vice versa, as per the requirement. A binary image can be obtained by the thresholding process. Global thresholding and local thresholding are the two classes of thresholding. ${ }^{21}$ In this work, global thresholding by Otsu's method is used and applied on the disc-removed image to segment blood vessels. Figure $8 \mathrm{~g}$ represents the binary image, consisting of blood vessels as the foreground white pixels. In addition to the blood vessels, a few small regions that are not part of the blood vessel pixels can be observed in this image. However, these small regions can be removed from the binary image by using morphological operations which open (push) the small isolated regions into the background (Fig. 8h). The effect of segmentation quality is therefore essential for achieving good segmentation results. There are many blood vessel segmentation techniques available in the previous works. We have used a simple threshold-based method that satisfies our requirements.

\subsection{Tortuosity measurement by counting the occurrence of vessel pixels}

To measure the tortuosity of a blood vessel, let us consider a binary image where blood vessels are represented by white pixels and the background by black pixels (Fig. 8h). From this binary image, some vessel segment regions are manually selected and cropped (Fig. 9a). This region is represented as a blood vessel segment image (Fig. 9b). This vessel segment image is then scanned from left to right, and the number of blood vessel pixels (white pixels) found in each scan is counted. This is repeated until scanning covers the entire vessel segment image. It is observed that, as vessel tortuosity increases, this process gives different results with increased number of vessel pixels.

To emphasize the effect of this observation, synthetically generated vessel segment images of size $100 \times 500$ were initially considered (Fig. 10). The vessel segments were classified into three types: normal, moderately tortuous, and severely tortuous. In our experiments, a normal vessel is considered to have fewer turns/twists compared to moderately tortuous and severely tortuous vessels. This observation is obtained from the literature and from the RET-TORT dataset. ${ }^{12}$

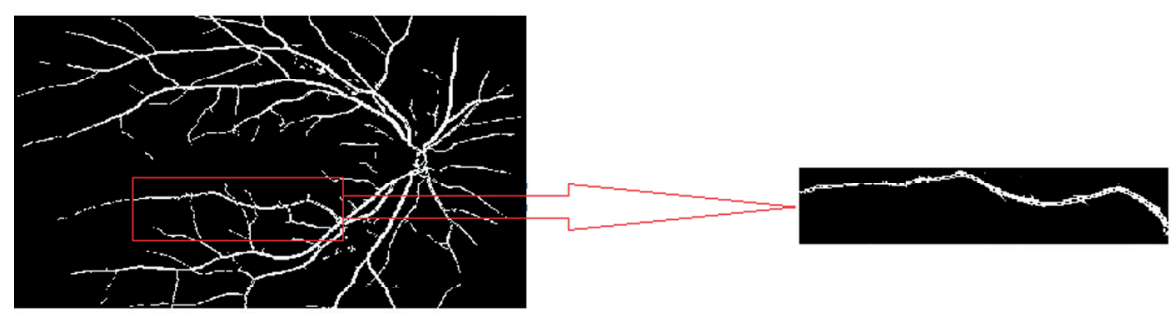

(a)

Fig. 9. (a) Blood vessel image. (b) Blood vessel segment image. 
Row 1 to 100

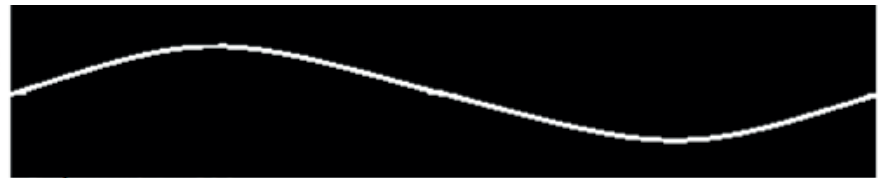

Column 1 to 500

(a)

Row 1 to 100

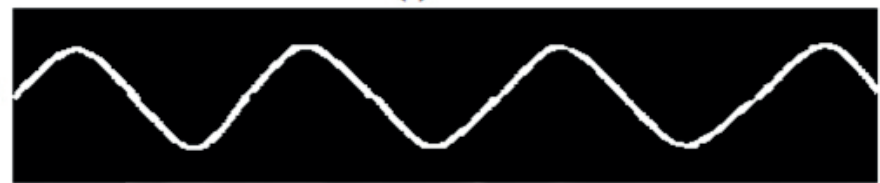

Column 1 to 500

(b)

Row 1 to 100

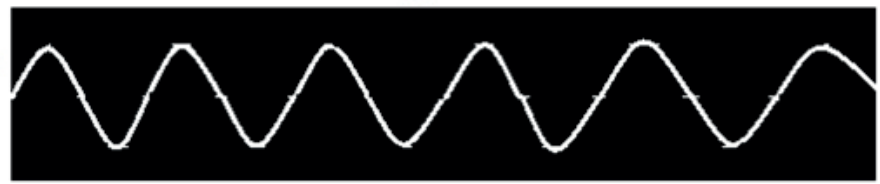

Column 1 to 500

(c)

Fig. 10. Synthetically generated $(a)$ normal, $(b)$ moderately tortuous, and $(c)$ severely tortuous vessel segment images.

The retinal vessel segment images in the RET-TORT database are arranged by a retinal specialist on the basis of increasing tortuosity by considering the number of turns/twists of the vessels. The vessels with fewer number of turns are given a low tortuosity ranking, whereas those with a greater number of turns are given a high tortuosity ranking. Thus, in our experiment we have also considered the number of turns/twists to classify the vessel segments as normal, moderately tortuous, or severely tortuous vessels.

For the synthetically generated vessels, the frequency of oscillations of the sine waves defines whether vessels are normal, moderately tortuous, or severely tortuous, given that the frequency of oscillations is related to the number of turns/ twists. Sine waves with a lower oscillation frequency are considered to have fewer number of turns or be gently curved; they are classified as normal vessels. Similarly, sine waves with a higher oscillation frequency are considered to have a greater number of turns; they are classified as moderately tortuous vessels. Vessels with a greater oscillation frequency than moderately tortuous vessels are classified as severely tortuous. Figure 10a-c shows increasing oscillation frequencies for normal, moderately tortuous, and severely tortuous vessel segments. Thus, the frequency of oscillation is considered as a parameter for classifying these vessel segments as normal, moderately tortuous, or severely tortuous. In our method, this is referred to as tortuosity of synthetically generated vessels. It should be noted that in all types of synthetically generated vessels, the amplitude of the sinusoids is the same 
while the oscillation frequency varies. Hence, we are considering that a synthetically generated normal vessel segment consists of a lower number of oscillations compared to moderately tortuous and severely tortuous vessel segments. The histograms showing the vessel pixel (white pixels) count for the corresponding synthetically generated blood vessels segments are shown in Figure 11a-c.

It can be observed from the graph in Figure 11a that the maximum occurrence of blood vessel pixels in a given row is five and that there are only two rows containing the maximum number of vessel pixels. The plot shows nearly a flat histogram, except for a few rows containing the maximum count. The histogram plot for a moderately tortuous vessel segment is shown in Figure $11 \mathrm{~b}$. In this case, the maximum occurrence of blood vessel pixels in a given row increases to 16 and the number of rows containing the maximum number of vessel pixels increases to 18 . The plot consists of more than one flat region. Figure 11c depicts a severely tortuous vessel segment, where the maximum occurrence of blood vessel pixels further increases to 24 and the number of rows having the maximum number of vessel pixels is 20 . In a similar fashion to the moderate case, the histogram consists of more than one flat region. The experiment is then repeated considering synthetically generated vessel segments of three different orientations: horizontal,
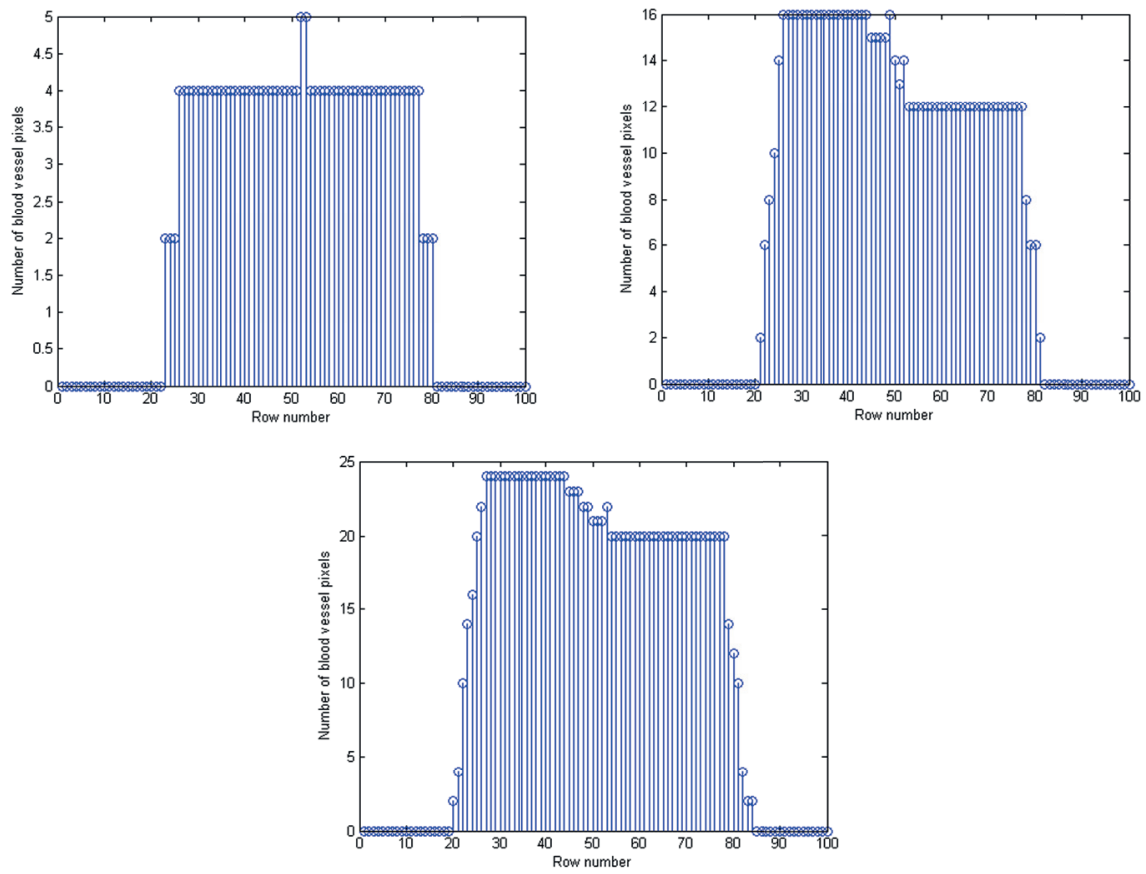

Fig. 11. Histogram of synthetically generated (a) normal, (b) moderately tortuous, and (c) severely tortuous vessel segments. 


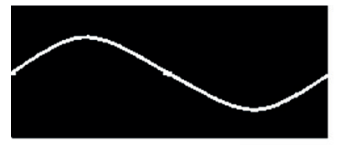

Normal ressel segment (hl)

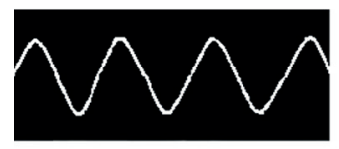

Moderately tortuous ressel segment (h2)

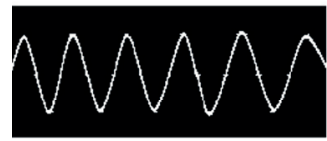

Severely tortuous vessel segment (h3)

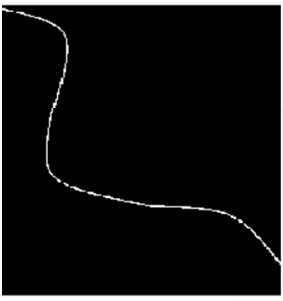

Normal ressel segment (dl)

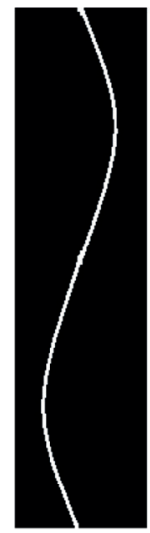

Normal vessel segment (v1)

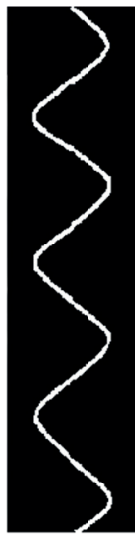

Moderately tortuous ressel segment $(\sqrt{2})$

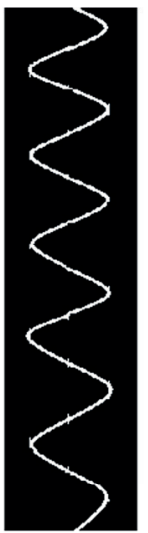

Severely tortuous ressel segment (v3)

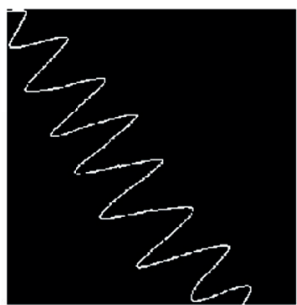

Severely tortuous ressel segment (d3)

Fig. 12. Synthetically generated blood vessel segments in different orientations .

vertical, and diagonal, as depicted in Figure 12. The sizes considered for horizontal, vertical, and diagonal segments are $100 \times 500,500 \times 100$, and $500 \times 500$ respectively. It is observed that, irrespective of orientation, the vessel pixel count increases with increasing oscillation frequency. This means that increasing tortuosity is reflected in an increased vessel pixel (white pixels) count. From these observations, we found that tortuosity can be defined as a combination of two parameters:

1. maximum number of vessel pixel occurrence (M); and

2. number of rows $(R)$ having vessel pixel occurrence $M$ and very close to $M$.

To explore the relevance of parameter $\mathrm{M}$ and parameter $\mathrm{R}$ in defining tortuosity, the method is applied on an additional nine synthetically generated horizontal vessel segments of size $100 \times 500$ (Fig. 13). Here, three normal (normal 1, normal 2, and normal 3), three moderately tortuous (moderate 1 , moderate 2 , and moderate 3 ), and three severely tortuous (severe 1 , severe 2 , and severe 3 ) vessel segments are taken into consideration so as to include variations in each case. In each of these images, the amplitude is the same, whereas the frequency of oscillation varies. Normal 2 and normal 3 differ from normal 1 in the sense that their oscillation frequency is slightly different. The oscillation frequency of normal 2 is slightly 


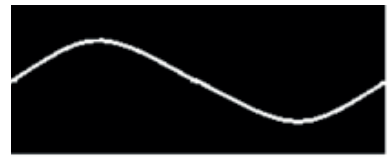

Normal 1

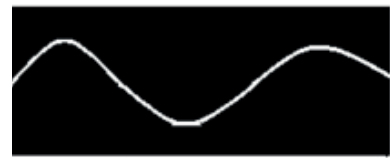

Normal 2

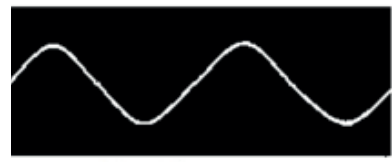

Normal 3

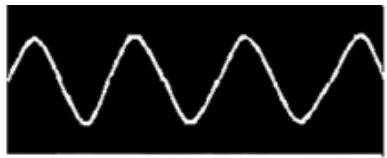

Moderate 1

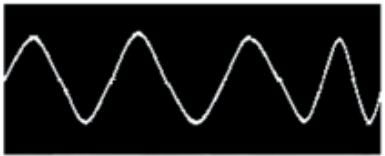

Moderate 2

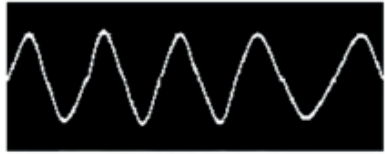

Moderate 3

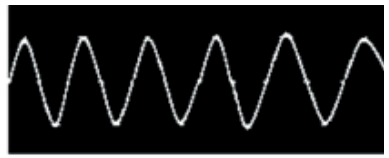

Severe 1

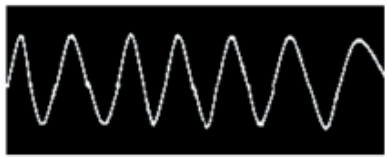

Severe 2

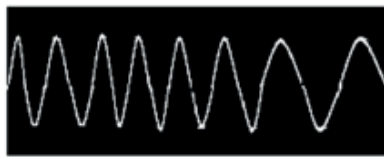

Severe 3

Fig. 13. Synthetically generated horizontal blood vessel segments of normal, moderate, and severe cases.

greater than normal 1, while normal 3 has an oscillation frequency slightly greater than normal 2 but less than moderate 1 . Similarly, in the case of moderately tortuous and severely tortuous vessel segments, oscillation frequency increases as we move from the top image to the bottom image (Fig. 13). A small overlap may sometimes exist between normal and moderate as well as moderate to severe case classifications. For instance, normal 3 may overlap (look similar) with moderate 1 , and moderate 3 may overlap with severe 1 . This requires us to consider the different types of normal, moderate, and severe vessels in our method. Subsequently, nine synthetically generated vertical and diagonal vessel segments, respectively, were also considered. Thus, a total of 27 vessel segments were considered for tortuosity evaluation and thereafter used in the experiment. The mean for parameter $\mathrm{M}$ and parameter $\mathrm{R}$ is obtained using 27 vessel segments.

In Figure 13, the size of all images is considered to be same. Now, in order to make the method work for blood vessels of different size, amplitude and orientation, four considerations are made for each type of synthetically generated horizontal vessel segments (normal, moderate, and severe) (Fig. 14). These four considerations are:

1. size is considered to be $100 \times 500$;

2. size is $100 \times 500$, but amplitude is increased;

3. size is now increased to $100 \times 600$, but amplitude is the same as in (1); and

4. size is again increased to $200 \times 700$, but amplitude is the same as in (1).

This gives a total of 12 considerations for horizontal blood vessel segments. Synthetically generated vessel segments with vertical and diagonal orientations were also considered. The mean values for parameter $\mathrm{M}$ and parameter $\mathrm{R}$ using the proposed method for tortuosity measurement after considering 36 (12 for 

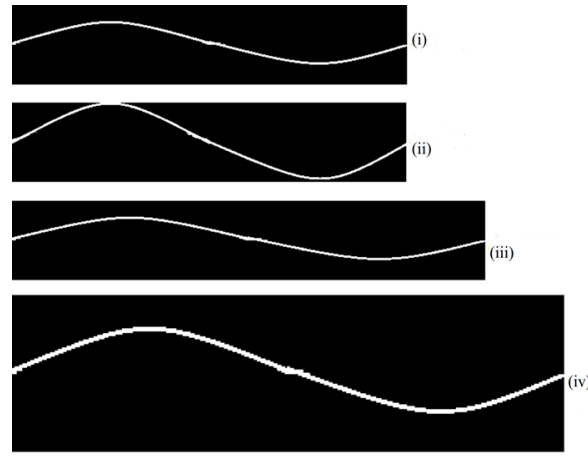
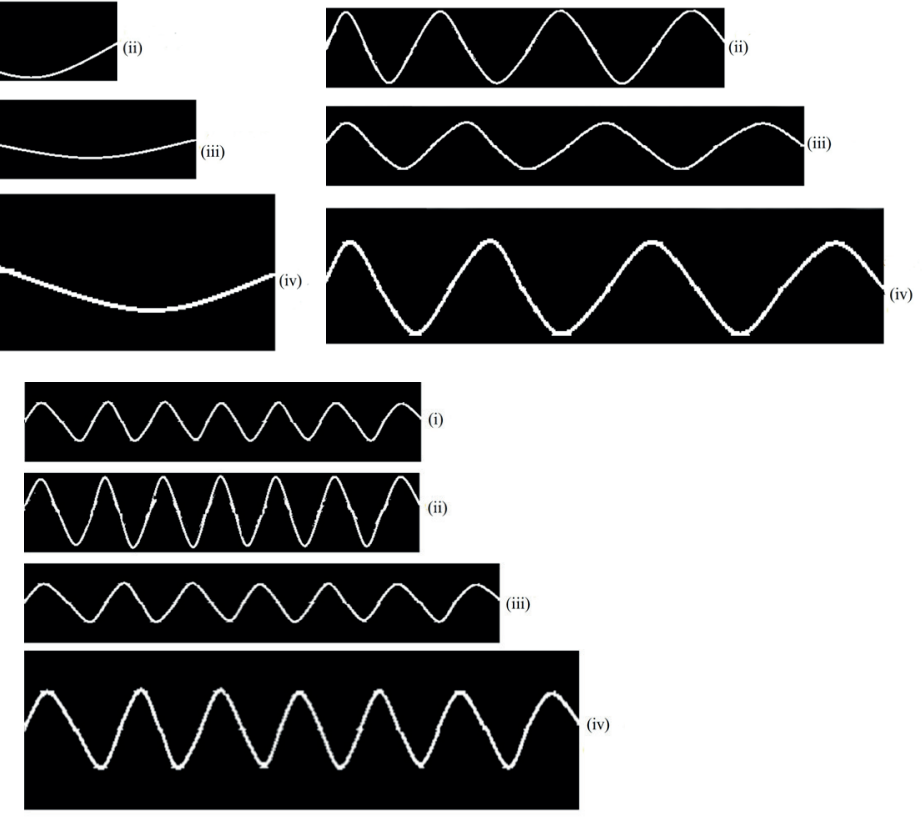

Fig. 14. Synthetically generated (a) normal, (b) moderately tortuous, and (c) severely tortuous horizontal vessel segments of varying amplitude and size.

horizontal, 12 for vertical, and 12 for diagonal vessel segments) synthetically generated vessel segments are noted down. The average values of $M$ and $R$ are calculated by considering the 27 and 36 numbers of vessel segments and are used for evaluating retinal vessel segment tortuosity. The results obtained from synthetically generated vessels thus constitute the baseline for evaluating the tortuosity of actual retinal blood vessels.

After applying the method on different synthetically generated vessel segments, the method was then applied to measure the tortuosity of actual retinal blood vessels. As different retinal images contain blood vessels of different amplitude, size, and orientation, the proposed method is applied to evaluate the tortuosity of retinal vessels. The method was first applied on 20 retinal images collected from the DRIVE database, ${ }^{17} 30$ retinal images collected from the HRF database, ${ }^{18}$ and 5 retinal images collected from a local eye hospital. These databases contain a large number of retinal images, thus allowing us to test the system to its full potential. ${ }^{22}$ For each of these images, five horizontal, five vertical, and five diagonal blood vessel segments were considered. This implies that, from a single retinal image, $15 \mathrm{blood}$ vessel (artery and vein) segments were considered. Therefore, the experiment was 


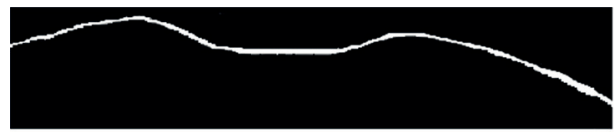

(a)

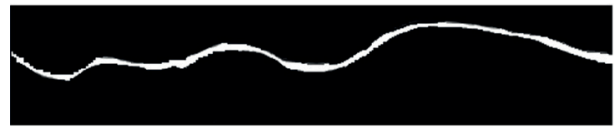

(b)

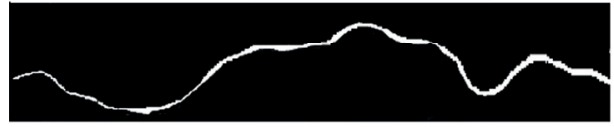

(c)

Fig. 15. Normal, (a) moderately tortuous (b), and severely tortuous $(c)$ retinal blood vessel segments oriented horizontally.
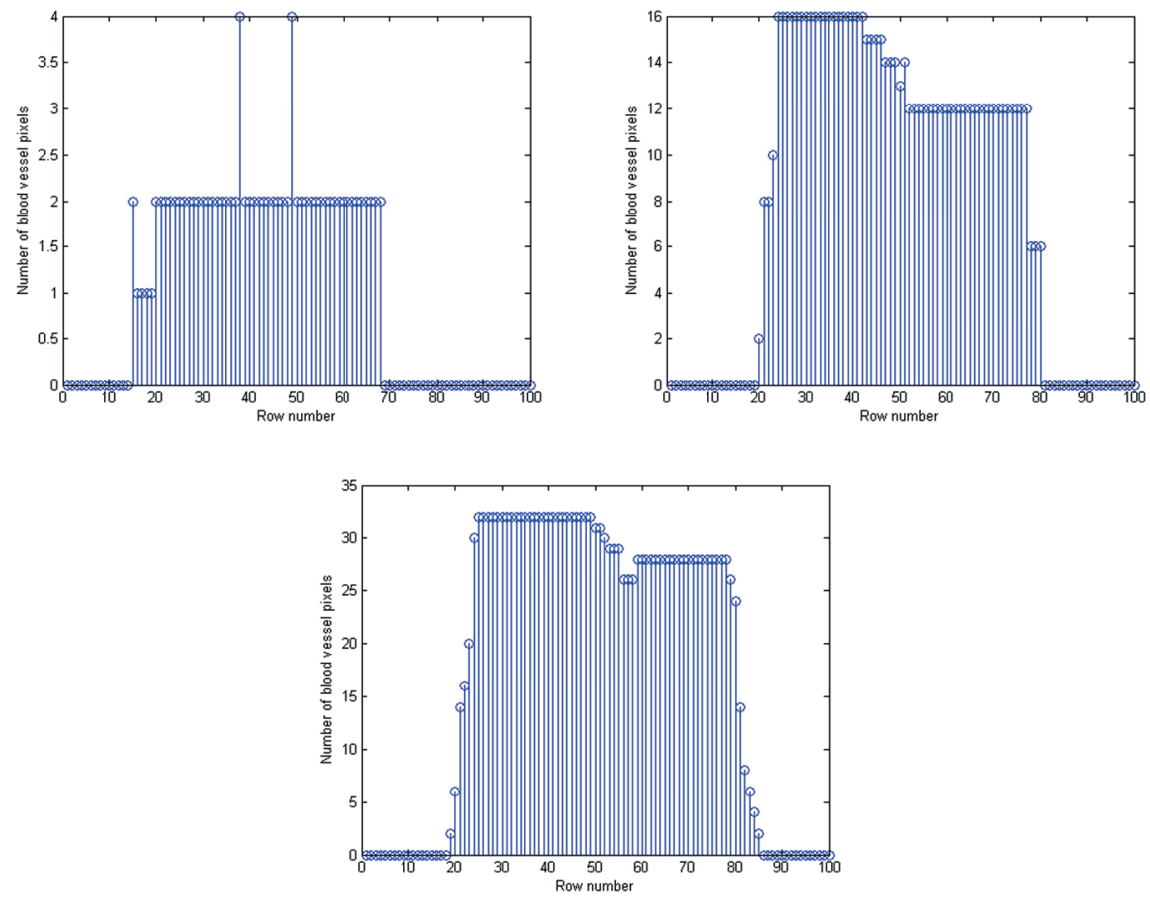

Fig. 16. Histogram of $(a)$ normal, (b) moderately tortuous, and (c) severely tortuous retinal blood vessel segments. 
performed on a total of 825 blood vessel segments to classify them as normal, moderately tortuous, or severely tortuous. Figure 15 illustrates a few horizontal retinal blood vessel image segments of a normal, moderately tortuous, and severely tortuous nature that were extracted from a retinal image. The corresponding histogram plot of blood vessel pixels is shown in Figure 16a-c. The results of tortuosity evaluation of retinal blood vessel segments using the proposed method is summarized in the Results section.

We would like to mention that we did not consider arteries and veins separately in the case of synthetically generated blood vessels, considering instead a general curve representing the blood vessel. We did, however, consider different types of synthetically generated vessel segments in relation to amplitude, size, and orientation. After conducting rigorous experiments on these synthetic vessels, the algorithm was applied to measure the tortuosity of actual retinal blood vessel segments containing both arteries and veins.

\subsection{Evaluation}

After applying the method on the retinal vessel segment images of the DRIVE ${ }^{17}$ and $\mathrm{HRF}^{18}$ database, the last stage of the proposed method is to evaluate the efficiency of our method in determining tortuosity. To do this, the retinal vessel segment images (30 arteries, 30 veins) from the RET-TORT database ${ }^{12}$ are used. The image size for arteries ranges from $78 \times 931$ to $266 \times 932$ and the image size for veins ranges from $52 \times 931$ to $259 \times 931$. All these images are provided with a clinical order number by a retinal specialist which represents the tortuosity level. It will be referred to as RET-TORT order in our method. By observing the vessel segments and their clinical order, these 30 vessel segment images are grouped into three categories:

1. RET-TORT order 1-11: Normal blood vessel (these vessel segments consist of fewer turns/twists);

2. RET-TORT order 12-27: Moderately tortuous blood vessel (number of turns/ twists are greater than normal but fewer than severely tortuous vessel); and

3. RET-TORT order 28-30: Severely tortuous blood vessel (number of turns/ twists are greater than moderately tortuous).

This grouping is done on the basis of visual examination of blood vessels from several images and based on the subjective opinion of different subjects. If the clinical ordering of the vessels and the results of the proposed method are similar, then it can be concluded that the proposed method has the capability of measuring the tortuosity of retinal blood vessels.

\section{Results}

The results for nine horizontal vessel segments (three normal, three moderately tortuous, and three severely tortuous), subsequently extended to nine vertical and 
Table 1. Mean of $\mathrm{M}$ and $\mathrm{R}$ for 27 synthetically generated blood vessel segments

\begin{tabular}{|l|l|l|l|}
\hline $\begin{array}{l}\text { Blood vessel } \\
\text { orientation }\end{array}$ & $\begin{array}{l}\text { Type of blood } \\
\text { vessel }\end{array}$ & Parameter $\mathbf{M}$ & Parameter R \\
\hline \multirow{4}{*}{ Horizontal } & Normal & 7 & 12 \\
\cline { 2 - 4 } & Moderate & 17 & 26 \\
\cline { 2 - 4 } Vertical & Severe & 28 & 21 \\
\hline \multirow{4}{*}{ Diagonal } & Normal & 7 & 9 \\
\cline { 2 - 4 } & Moderate & 17 & 21 \\
\cline { 2 - 4 } & Severe & 28 & 14 \\
\hline & Normal & 5 & 4 \\
\cline { 2 - 4 } & Moderate & 7 & 10 \\
\cline { 2 - 4 } & Severe & 8 & 5 \\
\hline
\end{tabular}

nine diagonal vessel segments (three normal, three moderately tortuous, and three severely tortuous, respectively), as shown in Figure 13, are presented in Table 1. For all vessel orientations - horizontal, vertical, and diagonal- parameter $\mathrm{M}$ and parameter $\mathrm{R}$ for normal, moderately tortuous, and severely tortuous vessels is obtained by taking the mean of three types of normal, three types of moderately tortuous, and three types of severely tortuous vessels, respectively. It is clear from Table 1 that the maximum vessel pixel count (M) increases from normal to moderately tortuous, and from moderately tortuous to severely tortuous vessels. The number of rows $(R)$ having the maximum count $(M)$ also increased as compared to normal vessels.

To eliminate bias in the method, the procedure was executed on 36 synthetically generated vessel segments of different size and amplitude, as shown in Figure 14. The results are presented in Table 2. The results illustrate that, irrespective of orientation, size, and amplitude, the tortuosity of the blood vessel segments increases with increasing oscillation frequency (curviness) of the blood vessel segments.

On taking the average of $\mathrm{M}$ and $\mathrm{R}$ from Table 1 and Table 2, the following conclusions can be drawn:

1. for normal vessels, ' $M$ ' and ' $R$ ' $\leq 10$

2. for moderately tortuous vessels, ' $M$ ' or ' $R$ ' fall in the range of 11-20; and

3. for severely tortuous vessels, ' $M$ ' or ' $R \geq 21$.

Applying these ranges to actual retinal blood vessels, we found that out of 825 retinal blood vessel segments, there are a total of 605 normal, 217 moderately tortuous, and 3 severely tortuous blood vessel segments. The results for parameters $M$ and $\mathrm{R}$, used for evaluating the tortuosity of retinal blood vessel segments employing the proposed method, are presented in Table 3. Parameters M and R for normal, 
Table 2. Mean of $\mathrm{M}$ and $\mathrm{R}$ for 36 synthetically generated blood vessel segments after considering the four cases

\begin{tabular}{|l|l|l|l|}
\hline $\begin{array}{l}\text { Blood vessel } \\
\text { orientation }\end{array}$ & $\begin{array}{l}\text { Type of blood } \\
\text { vessel }\end{array}$ & Parameter M & Parameter R \\
\hline \multirow{4}{*}{ Horizontal } & Normal & 5 & 15 \\
\cline { 2 - 4 } & Moderate & 16 & 14 \\
\cline { 2 - 4 } & Severe & 28 & 17 \\
\hline \multirow{4}{*}{ Vertical } & Normal & 8 & 15 \\
\cline { 2 - 4 } & Moderate & 12 & 24 \\
\hline \multirow{4}{*}{ Diagonal } & Severe & 24 & 33 \\
\hline & Normal & 5 & 3 \\
\cline { 2 - 4 } & Moderate & 8 & 5 \\
\cline { 2 - 4 } & Severe & 9 & 5 \\
\hline
\end{tabular}

Table 3. Tortuosity of retinal blood vessel segments using the proposed method

\begin{tabular}{|l|l|l|l|}
\hline $\begin{array}{l}\text { Blood vessel } \\
\text { orientation }\end{array}$ & $\begin{array}{l}\text { Type of blood } \\
\text { vessel }\end{array}$ & Parameter M & Parameter $\mathbf{R}$ \\
\hline \multirow{4}{*}{ Horizontal } & Normal & 8 & 5 \\
\cline { 2 - 4 } & Moderate & 13 & 6 \\
\cline { 2 - 4 } & Severe & 22 & 10 \\
\hline \multirow{4}{*}{ Vertical } & Normal & 8 & 5 \\
\hline \multirow{3}{*}{ Diagonal } & Moderate & 13 & 5 \\
\cline { 2 - 4 } & Severe & 21 & 10 \\
\hline & Normal & 6 & 5 \\
\cline { 2 - 4 } & Moderate & 7 & 10 \\
\cline { 2 - 4 } & Severe & 8 & 12 \\
\hline
\end{tabular}

moderately tortuous, and severely tortuous vessels oriented in horizontal, vertical and diagonal directions are obtained by finding the average for all normal, moderately tortuous, and severely tortuous vessels, respectively. These results were verified by conducting subjective evaluations, as discussed in the next section.

To check the validity of our method, the method was evaluated by comparing with the vessel segment images in the RET-TORT database. ${ }^{12}$ The evaluation of the 
Table 4. Comparison between RET-TORT order and proposed method

\begin{tabular}{|c|c|c|c|c|}
\hline $\begin{array}{l}\text { RET-TORT } \\
\text { order }\end{array}$ & $\begin{array}{l}\text { Visual } \\
\text { examination }\end{array}$ & $\begin{array}{l}\text { 'M' of the } \\
\text { proposed } \\
\text { method }\end{array}$ & $\begin{array}{l}\text { ' } R \text { ' of the } \\
\text { proposed } \\
\text { method }\end{array}$ & $\begin{array}{l}\text { Remark of } \\
\text { the proposed } \\
\text { method }\end{array}$ \\
\hline 1 & Normal & 6 & 7 & Normal \\
\hline 2 & Normal & 8 & 1 & Normal \\
\hline 3 & Normal & 10 & 1 & Normal \\
\hline 4 & Normal & 10 & 1 & Normal \\
\hline 5 & Normal & 10 & 2 & Normal \\
\hline 6 & Normal & 10 & 1 & Normal \\
\hline 12 & Moderate & 12 & 3 & Moderate \\
\hline 13 & Moderate & 14 & 3 & Moderate \\
\hline 14 & Moderate & 20 & 4 & Moderate \\
\hline 15 & Moderate & 16 & 4 & Moderate \\
\hline 16 & Moderate & 14 & 8 & Moderate \\
\hline 17 & Moderate & 18 & 3 & Moderate \\
\hline 28 & Severe & 19 & 8 & Moderate \\
\hline 29 & Severe & 18 & 7 & Moderate \\
\hline 30 & Severe & 18 & 9 & Moderate \\
\hline
\end{tabular}

proposed method closely follows the clinical ordering of vessels as provided by the RET-TORT database. A number of results showing a comparison between the ranking of RET-TORT vessel segment images using our proposed method and the clinical ordering by the retinal specialist in the RET-TORT database are presented in Table 4. However, it was found that, in some cases, the method was not able to classify severe cases correctly, classifying them instead as moderate. Nonetheless, normal cases were treated correctly. Table 4 shows that, although severe cases were classified as moderately tortuous by the proposed method, the values of parameter $\mathrm{M}$ and parameter $\mathrm{R}$ lie close to the upper limit of moderately tortuous blood vessels ( $M$ or $R$ close to 20). This shows that there is a small overlap between normal to moderate and moderate to severe case classifications.

The experimental results show that the proposed method evaluates tortuosity efficiently and vessels are classified accordingly into normal, moderately tortuous and severely tortuous. 


\section{Subjective evaluation}

To investigate the effectiveness of the proposed method, the tortuosity of different retinal vessels used in the method were also evaluated subjectively. Three postgraduate students and three research scholars working in the field of retinal image processing subjectively evaluated the tortuosity of blood vessel segments. Blood vessels from ten retinal images belonging to the HRF database were cropped out manually and displayed to the subjects as normal, moderately tortuous, and severely tortuous according to the number of twists and turns. The subjects were thus initially trained to identify normal and tortuous blood vessels and asked to provide an opinion score of 1 for normal, 2 for moderately tortuous, and 3 for severely tortuous blood vessels. The 825 blood vessel segment images were then arranged randomly to assess the ability of the subjects in identifying the blood vessels. The mean opinion score (MOS) was calculated for each image and a graph was plotted (Fig. 17). The subjective results state that out of those 825 blood vessel segments, 610 were normal, 210 were moderately tortuous, and 5 were severely tortuous blood vessel segments. This indicates that the method results correlate closely with the subjective evaluation.

\section{Discussion}

The method presented in this paper can be effectively used to evaluate retinal blood vessel tortuosity. The tortuosity evaluation using synthetically generated vessel segments shows promising results. The range of values for parameters $\mathrm{M}$ and $R$ were obtained from these experiments and thus used as a baseline for measuring retinal blood vessel tortuosity. When the method is applied to actual retinal blood vessel segments obtained from retinal images of publicly available databases, the results obtained show that vessels are classified as normal, moderately tortuous, and severely tortuous. While many of the existing tortuosity evaluation methods have not been applied to retinal vessel segments, our method was tested on artery and vein retinal vessel segments. Furthermore, only a limited number of methods have classified the vessels, but the databases used in the work are not publicly available. ${ }^{11,15}$ In our method, most of the images were obtained from publicly available databases. The results of our method were also compared with the clinical ordering of images in the RET-TORT database. The experimental results and the clinical ordering of images provided by the retinal specialist in the RET-TORT database as well as the results of subjective evaluation closely match and hence, the proposed method can be efficiently used for evaluating the tortuosity of blood vessels. However, we have observed the following limitations in the proposed method:

1. There is an overlap in classification between normal and moderately 


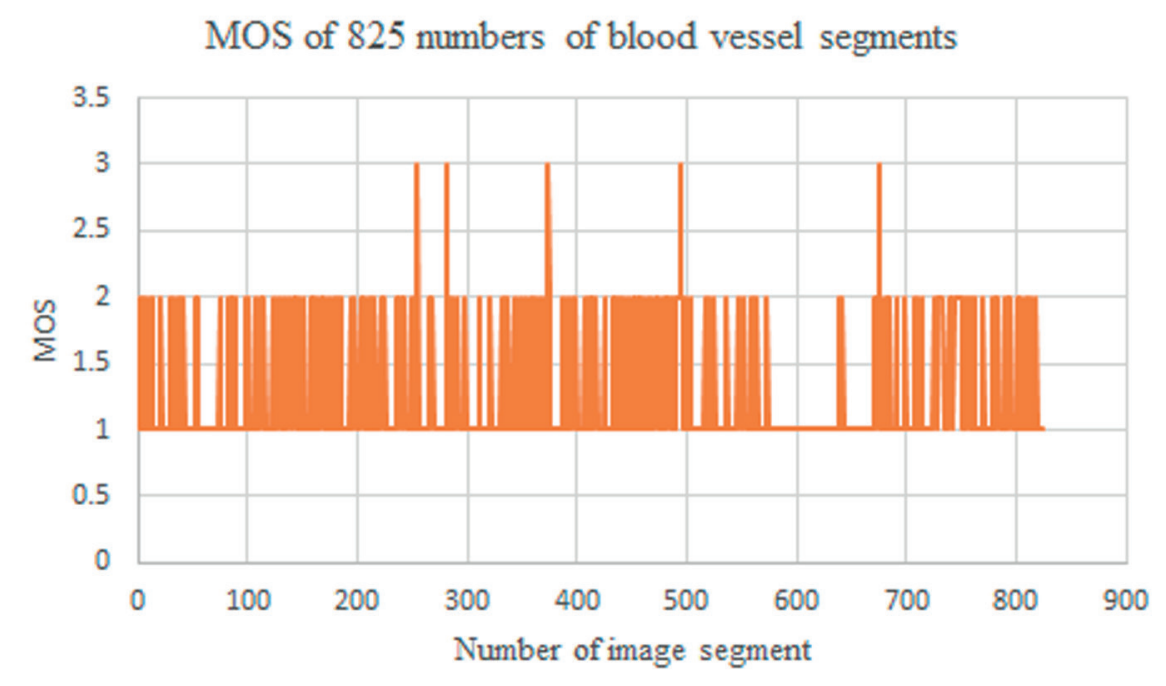

Fig. 17. MOS graph from the 6 subjects for 825 blood vessel segments.

tortuous blood vessels, and between moderately tortuous and severely tortuous blood vessels.

2. We have used a simple threshold-based image segmentation technique; nonetheless, the segmentation results were sufficient for performing our method.

We observed that, in a few images, some unwanted regions that were not part of the vessels remained after the segmentation step, which may have affected our results to some extent. Thus, improving segmentation quality is essential for obtaining better results.

\section{Conclusion}

The aim of this study is to measure the tortuosity of retinal blood vessels, which may help in detecting hypertensive retinopathy. We propose a simple but efficient image processing-based method to measure the tortuosity of retinal blood vessels. We found that in binary blood vessel images, the vessel pixel (white pixels) count increases with increasing vessel tortuosity. Two parameters were used to evaluate tortuosity. One is parameter $\mathrm{M}$, which represents the maximum vessel pixels; the other is parameter $\mathrm{R}$, which is described as the number of rows having $M$ vessel pixels. The values of parameters $M$ and $R$ increase from normal to moderately tortuous, and from moderately tortuous to severely tortuous vessels. When the method was applied to the actual retinal blood vessel segments, the results obtained shows that 
vessels are classified as normal, moderately tortuous, and severely tortuous. The method was also applied on tortuous vessel database (RET-TORT) images and the measured tortuosity level closely matches with the expert ranking. Similarly, there was a strong correlation between retinal vessel classification using the proposed method and retinal vessel classification performed by different subjects. Our future work will focus on testing the entire blood vessel instead of considering only segments and then classifying them as normal or tortuous. Given that very few tortuous images were considered in the work, we also plan to collect hypertensive retinopathy images in order to apply the method on them.

\section{Acknowledgements}

The authors would like to acknowledge Dr. Narayan Bardoloi, Medical Director of Chandraprabha Eye Hospital (Jorhat, Assam, India) for providing the necessary materials and giving his valuable suggestions and guidance for carrying out this research work.

\section{References}

1. Wang X, Cao H, Zhang J. Analysis of retinal images associated with hypertension and diabetes. In: Engineering in Medicine and Biology Society, 2005. IEEE-EMBS 2005. 27th Annual International Conference of the 2006 Jan 17 (pp. 6407-6410). doi:10.1109/IEMBS.2005.1615964.

2. Talu S. Characterization of retinal vessel networks in human retinal imagery using quantitative descriptors. Human and Veterinary Medicine. 2013;5(2):52-57.

3. Bhargava M, Wong TY. Current concepts in hypertensive retinopathy. Retinal Physician; 2013. Available from: https://www.retinalphysician.com/issues/2013/nov-dec/current-concepts-in-hypertensive-retinopathy

4. Wong TY, McIntosh R. Hypertensive retinopathy signs as risk indicators of cardiovascular morbidity and mortality. Br Med Bull. 2005 Sep 7;73-74 (1):57-70. doi:10.1093/bmb/ldh050.

5. Dougherty G, Varro J. A quantitative index for the measurement of the tortuosity of blood vessels. Med Eng Phys. 2000;22(8):567-574.

6. Oh KT. Ophthalmologic manifestations of hypertension, 2016. Available from: https://emedicine. medscape.com/article/1201779-overview.

7. Patasius M, Marozas V, Lukosevicius A, Jegelevicius D. Model based investigation of retinal vessel tortuosity as a function of blood pressure: preliminary results. In: Engineering in Medicine and Biology Society, 2007. EMBS 2007. 29th Annual International Conference of the IEEE 2007 Aug 22 (pp. 64596462). doi:10.1109/IEMBS.2007.4353838

8. Mondal RN, Martin MA, Rani M, et al. Prevalence and risk factors of hypertensive retinopathy in hypertensive patients. J Hypertens. 2017;6(2):1-5. doi:10.4172/2167-1095.1000241

9. Duncan BB, Wong TY, Tyroler HA, Davis CE, Fuchs FD. Hypertensive retinopathy and incident coronary heart disease in high risk men. Br J Ophthalmol. 2002;86(9):1002-1006.

10. Ong YT, Wong TY, Klein R, et al. Hypertensive retinopathy and risk of stroke. Hypertension. 2013 Oct;62(4):706-11. doi:10.1161/HYPERTENSIONAHA.113.01414. 
11. Sharbaf MA, Pourreza HR, Banaee T. A novel curvature-based algorithm for automatic grading of retinal blood vessel tortuosity. IEEE IEEE J Biomed Health Inform. 2016;20(2):586-595. doi:10.1109/ JBHI.2015.2396198.

12. BiolmLab-Laboratory of Biomedical Imaging- Retinal Vessel Tortuosity Data Set. Available from: http://bioimlab.dei.unipd.it/Retinal\%20Vessel\%20Tortuosity.htm

13. Grisan E, Foracchia M, Ruggeri A. A novel method for the automatic grading of retinal vessel tortuosity. IEEE Trans Med Imaging. 2008;27(3):310-319. doi:10.1109/TMI.2007.904657.

14. Cavallari M, Stamile C, Umeton R, Calimeri F, Orzi F. Novel method for automated analysis of retinal images: results in subjects with hypertensive retinopathy and CADASIL. Biomed Res Int. 2015;2015:752957. doi: 10.1155/2015/752957.

15. Turior R, Onkaew D, Uyyanonvara B, Chutinantvarodom P, Orzi F. Quantification and classification of retinal vessel tortuosity. ScienceAsia. 2013;39(3):265-277. doi:10.2306/scienceasia1513-1874.2013.39.265

16. El Abbadi NK, Al Saadi EH. Automatic retinal vessel tortuosity measurement. Journal of Computer Science. 2013;9(11):1456-1460. doi: 10.3844/jcssp.

17. DRIVE database. Available from: http://www.isi.uu.nl/Research/Databases/DRIVE.

18. High-Resolution Fundus (HRF) Image Database. Available from: https://www5.cs.fau.de/research/ data/fundus-images

19. Shome SK, Vadali SRK. Enhancement of diabetic retinopathy imagery using contrast limited adaptive histogram equalization. International Journal of Computer Science and Information Technologies. (2011;2(6):2694-2699.

20. Umbaugh S. Computer vision and image processing. New Jersey: Prentice Hall; 1998.

21. Singh TR, Roy S, Singh OI, Sinam T, Singh KM. A New adaptive thresholding technique in binarization. International Journal of Computer Science Issues. 2011;8(6):271-277.

22. Chetia S, Nirmala SR. Retinal blood vessel tortuosity measurement for analysis of hypertensive retinopathy. In: Innovations in Electronics, Signal Processing and Communication (IESC), 2017 International Conference on 2017 Apr 6 (pp. 45-50). doi:10.1109/IESPC.2017.8071862 